\title{
提高配电网的功率因数, 优化无功配置
}

\section{Improving Power Factor of Distribution Network and Optimizing Reactive Power Allocation \\ 李倩}

Qian Li

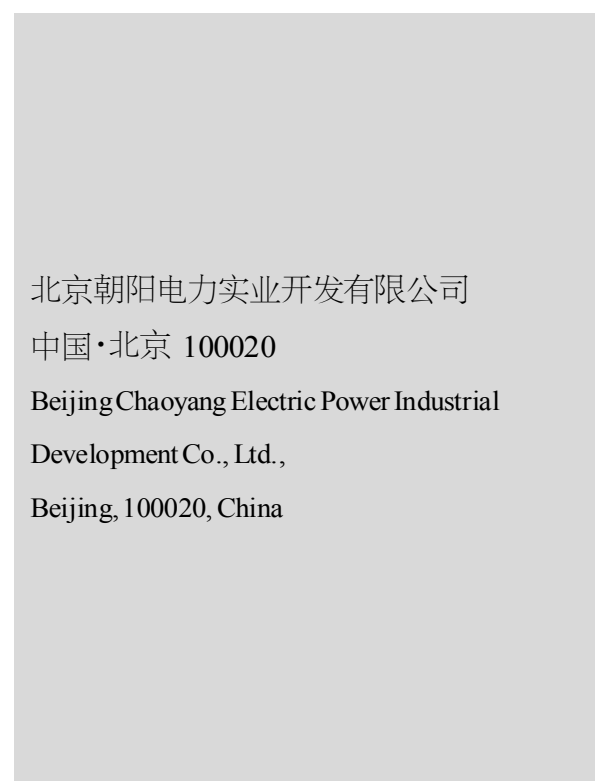

【摘要】目前, 许多企业所使用的电力系统中, 企业在电能和设备上的投入与所带来的 经济严重不符。除去电力系统保障的基本生产之外, 有许多不必要的电力损失, 众多因素 中无功负荷所产生的不良影响最为严重。因此, 为解决这一普遍问题, 便需要优化无功配 置, 进行相关的无功补偿来提高配电网的功率因数。

【Abstract】At present, in the power system used by many enterprises, the investment in electric energy and equipment by enterprises is seriously inconsistent with the economy brought about by them. In addition to the basic production of power system security, there are many unnecessary power losses. Among many factors, reactive power load has the most serious adverse impact. Therefore, in order to solve this common problem, it is necessary to optimize the reactive power allocation, and to improve the power factor of distribution network through reactive power compensation.

【关键词】配电网;功率因数; 优化无功配置

【Keywords】distributionnetwork; power factor; optimal reactive power allocation 【DOI】10.36012/peti.v1i1.357

\section{1 引言}

电力为中国近年来飞速发展进程中的一块基石, 与其相 关的电力系统便十分重要。然而, 现阶段大部分的电力系统中 都存在着电能利用率低下、无功电荷较多等致命缺点, 这不仅 仅是对电能的一种浪费, 而且会对相关配电网造成损坏。在大 部分的电力系统中, 一部分电力进行相关的有效输送时, 会流 失另一部分电力作为损耗, 这种损耗源于各种方面, 如配电变 压器、用电的设备、配电网、配电线路等。为了减少这种无功电 荷, 减少电力在电网的输送过程中损失的电力, 可以通过提高 配电网的功率因数、优化无功配置等多方面进行优化。对电力 系统的优化势在必行, 这既可以提高电力的使用效率, 减少配 电网的损坏率, 又可以增加相关输送网的耐久性及输送能力, 进而提高相关电力的实际利用率 ${ }^{[1]}$ 。

\section{2 功率因数必须提高的原因}

生活中见到的部分用电设备的功率因数都是小于 1 的, 如爱迪生所发明的白炽灯(现已被禁止使用)、电阻、使用电热
的相关设备。对于那些功率因数小于 1 的设备, 在轻载或空载 的情况下, 功率因数会随着设备所载负荷的降低而降低, 这不 仅会对相关单位造成不必要的经济损失, 而且还会令相关设 备的生命周期大幅缩短, 损坏设备, 具体分析如下:

(1)相关电气设备不能发挥出应有的能力, 不能创造出实 际效益。以发电机为例, 每一台发电机都具有额定电压与额定 电流, 在使用的过程中这都是不允许被超过的, 否则可能会导 致设备的损坏。现在还没有效率达到 $100 \%$ 的设备,所以在发 电机运转的过程中, 除了供其运转的基本电力之外, 还要损失 一部分的电力, 而当发电机轻载时, 功率因数会降低并且导致 实际功率的大幅度下降, 所以, 为了提高实际功率, 必须提高功 率因数, 以确保相关电气设备实际使用率达到最优。

(2)电能损耗增加,则企业经济效益大幅度减少。当设备的 功率因数降低时, 为了达到目标值, 要么使用更多的相同设备 来进行同时输出, 要么就需要增加多条线路进行输送, 二者的 目的都是一样的,减少因功率因数过低而带来的损失。但是, 这便会增加电流的输送量, 输送方式增加, 在损耗更多电能的 
同时还会对线路造成损坏, 对设备造成损坏, 得不偿失。尤其 是在当下这种社会阶段, 大部分地区经常会出现用电高峰期, 功率因数的降低不仅仅是一台设备的损失, 更会对大部分生 产造成直接经济损失 ${ }^{[2]}$ 。

\section{3 提高功率因数的方法}

由上述功率因数过低所引发的问题可得: 提高功率因数, 优化无功配置。必须仔细研究, 认真对待, 想出办法对其进行 解决。以下主要对减少电力系统中负载的无功损耗及优化其 无功配置并对其进行无功补偿两个点进行详细分析, 以供读 者参考。

\section{1 减少电力系统中负载的无功功率损耗}

减少无功功率的损耗主要以提高自然功率因数为主, 改 良用电设备及输出端设备为辅来达到目的。

(1)改良用电设备合理调整用电的电压比, 对部分用电设 备进行相关方面的改进、优化。在生产制造的过程中, 应增加 相关技术人员, 对相关的技术人员进行系统科学的培训, 在设 备使用一段时间之后安排维护检修，检查设备是否正常运转。 对于一些设备来说, 只要进行正常的运转, 损耗的无功功率已 经通过生产人员相关的技术操作降到了最低。但当这种设备在 使用一段时间之后若出现了问题，那么线路的损坏就会变大， 设备无法正常运转, 无功功率的损耗很可能远远超出正常值, 所以,改良用电设备并对设备进行定期的维护意义重大。

(2)合理选择输出端, 下面以变压器为例进行分析。变压器 的工作是通过电磁转换进行的, 在电磁转换的过程中, 如果负 载控制, 会损耗很多的无功功率, 造成不必要的损失。变压器 的功率因数分为两个步骤进行分析, 变压器第一次的功率因 数通过负载电荷可得出, 即随着负载电荷的变化而变化且成 正比。因此, 要保证负荷的合理程度。变压器第二次功率因数 是在已经包含了负载电荷所产生影响的前提下进行二次计 算。若想变压器的一次功率因数增加, 必须增加负荷, 而当变 压器空载时, 功率因数会降到最低, 实际效率降到最低。基于 此,为了保证生产的最大利益化、最大经济化, 目前所使用变 压器的过程中, 变压器的负荷功率达到 50\%时最优, 虽然变 压器功率因数随着负荷的变化而变化, 但是当负荷变得过大 时 (通常指超过 $50 \%$ ), 会对变压器造成损坏, 反而达不到预 期效果 ${ }^{[3]}$ 。

\section{2 优化无功配置, 进行无功补偿}

\section{2 .1 非人工式的无功补偿}

在进行无功补偿的过程中, 主要目的是为了减少无功功 率在配电网中的输送, 减少多余电荷的流动, 而减少的负荷大
部分被称为感性负荷。感性负荷在电网的流动过程中会交换 无功功率、吸收无功功率, 并且还会对有功功率进行损耗, 大 幅度地降低功率因数。像前文所提到的变压器、发电机还有电 动机等, 配电网对其的电力负荷主要为感性负荷, 通过改善配 电网的运输条件, 优化配电网的配置及设计, 可以较大程度上 降低感性负荷所带来的影响, 进行无功补偿降低功率因数。为 解决感性负荷吸收有功功率这一问题, 可以在系统中引入具 有容量且可以提供能量的负荷, 即容性负荷。当两种负荷在配 电网系统中进行流动时, 容性负荷因为本身就具有特殊性, 所 以, 对有功功率产生的影响微不足道, 可以忽略不计。此外, 当 感性负荷需要通过消耗有功功率来满足自身所需要的能量 时, 容性负荷可以通过释放自身所具有的能量来令其满足。这 样通过容性负荷和感性负荷两者之间在配电网输送的过程中 的不断交换与吸收, 可以大幅度提升有功功率并且降低无功 功率, 减少感性负荷对无功功率的交换与吸收 ${ }^{[4]}$ 。

\section{2 .2 人工式的无功补偿}

除却上述通过非人工补偿来提高配电网的功率因数之 外, 还可以通过人工的方式优化无功配置并人工进行无功补 偿。用电设备运转过程中所需要的无功功率通过人工计算后 由相关设备进行无功补偿, 如同步电动机、移相电容器、同步 调相机等多种无功补偿设备。这些无功补偿设备可以对运转 过程中的设备进行无功补偿, 使其无功功率通过外界获得而 不必再去通过消耗自身的有功功率来维持正常运转。虽然成 本较高, 但可持续使用, 且可以一定程度上提高了配电网的功 率因数。

\section{4 结语}

本文就提高配电网的功率因数、优化无功配置进行分析, 首先, 围绕在设备使用过程中功率因数过低会对相关人员及 设备造成的严重影响进行概述, 体现出提高功率因数的重要 意义; 其次, 对提高功率因数可以使用的两种主要方法进行概 述, 为读者提供两种主要参考方式; 最后, 对两种方法进行剖 析介绍, 表现两种提高功率因数方法的可行性与优越性。优化 无功配置、提高配电网的功率因数势在必行。

\section{参考文献}

[1]张丽华.中低压配电网的无功补偿优化[].城市建设理论研究, 2015(32):1339-1340.

[2]傅军栋, 喻勇,黄来福. 不平衡负载的一种更加经济的补偿方法 [J].电力系统保护与控制,2015(2):126-132.

[3]刘建辰,刘山林.基于二阶锥松驰和 Big-M 法的配电网分布式 电源优化配置[].电网技术,2018(8):2604-2611.

[4]张国平.配电网无功补偿优化探析[J].电工技术,2017(4):148-150. 